TITRE: LA FAMILLE SEIGNEURIALE RIOUX À TROIS-PISTOLES : UN ENRACINEMENT PORTEUR D'UNE MÉMOIRE PERSISTANTE

AUTEUR: NICHOLAS THÉROUX, CONSEILLER À L'INNOVATION ET À LA RECHERCHE À L'INSTITUT NATIONAL DES MINES ET CANDIDAT À LA MAîTRISE EN ADMINISTRATION PUBLIQUE À L'ÉCOLE NATIONALE D'ADMINISTRATION PUBLIQUE (ENAP)

PublicATION: LE RÉGIME SEIGNEURIAL AU QUÉBEC : FRAGMENTS D’HISTOIRE ET DE MÉMOIRE

PAGES: $24-53$

DIRECTION : BENOÎT GRENIER AVEC LA COLLABORATION D'AlAIN LABERGE ET DE STÉPHANIE LANTHIER

ISBN: 978-2-7622-0361-5

URI: HTTP://HDL.HANDLE.NET/11143/16418

DOI: HTTPS://DOI.ORG/10.17118/11143/16418 


\section{LA FAMILLE SEIGNEURIALE RIOUX À TROIS-PISTOLES : UN ENRACINEMENT PORTEUR D’UNE MÉMOIRE PERSISTANTE}

Nicholas Théroux, Conseiller à l'innovation et à la recherche à l'Institut national des mines et candidat à la maîtrise en administration publique à l'École nationale d'administration publique (ENAP)

La seigneurie de Trois-Pistoles se situe dans l'actuelle région du Bas-Saint-Laurent, c'està-dire sur la rive sud du fleuve Saint-Laurent à l'est de Québec. La concession de cette seigneurie située dans le gouvernement de Québec se déroule en 1687 lorsque le gouverneur Brisay de Denonville et l'intendant Bochart de Champigny concèdent à Charles Denys de Vitré un fief de « deux lieues de front le long du fleuve Saint-Laurent ${ }^{1}$ » à prendre à partir de la fin de la seigneurie de l'île-Verte. Actif dans le domaine de la pêche et siégeant au Conseil souverain, de Vitré ne concentre pas d'efforts dans la colonisation de sa seigneurie puisqu'il entend plutôt utiliser son territoire comme un point d'appui pour ses activités de pêche sur le Saint-Laurent². Loin d'être son seul fief, Trois-Pistoles fait au contraire partie intégrante de l'ensemble seigneurial qui repose entre les mains de ce seigneur (celui-ci détient notamment les seigneuries du Bic et de Bellevue) ${ }^{3}$.

L’année 1696 marque cependant un tournant majeur pour la seigneurie puisque celle-ci passe entre les mains de Jean Rioux ${ }^{4}$. Cet habitant de l'île d'Orléans échange en effet sa censive défrichée contre la seigneurie en «bois debout » possédée par de Vitré5. Cette accession à la propriété seigneuriale de la part d'un paysan peut sembler étonnante à priori, mais la situation géographique défavorisée du fief (celui-ci est excentré par rapport aux centres urbains autour desquels se développe principalement la colonisation) permet de comprendre plus aisément les motifs de cet échange impliquant une censive bien située (et mise en valeur) en contrepartie d'une seigneurie établie sur les terres les « moins attrayantes du ter-

1. Société d'histoire et de généalogie de Trois-Pistoles, Fonds Anita-Rioux, Acte de concession de la seigneurie de Trois-Pistoles, le 6 janvier 1687.

2. Jean-Charles Fortin et Antonio Lechasseur, Histoire du Bas-Saint-Laurent, Québec, Institut québécois de recherche sur la culture, 1993, p. 122.

3. A. J. E Lunn, «Charles Denys de Vitré », Dictionnaire biographique du Canada » [en ligne], Consulté le 29 janvier 2018, http://www.biographi.ca/fr/bio/denys_de_vitre_charles_2F.html

4. Alain Laberge, Portraits de campagnes : la formation du monde rural laurentien au XVIIIe siècle, Québec, Presses de I'Université Laval, 2010, p. 83.

5. Benoît Grenier, Brève histoire du régime seigneurial, Montréal, Boréal, 2012, p. 122. 
ritoire laurentien ${ }^{6} »$. D'ailleurs, ce type d'échange ne constitue pas une opération inusitée en ce tournant de siècle puisque deux autres familles paysannes de l'île d'Orléans réalisent à cette époque le même type d'échange que les Rioux. En effet, les Lepage et les Côté se retrouvent également à la tête d'une seigneurie située dans l'actuel Bas-Saint-Laurent en 1694 et en $1711^{7}$. Comme plusieurs autres seigneurs d'origines modestes, Jean Rioux entreprend lui-même d'entamer l'exploitation de son fief, ce qui fait de lui l'un des rares véritables « seigneurs-défricheurs » de la Nouvelle-France ${ }^{8}$. À la mort du seigneur Jean Rioux en 1709, le fief ne compte toujours aucun censitaire ${ }^{9}$. Comme le souligne Valérie Nicolas, cet état des choses découle en partie de « l'éloignement de la seigneurie de Trois-Pistoles [des] centres importants et [de] la déficience des voies de communication » permettant d'accéder à celle$\mathrm{Ci}^{10}$. Il faudra attendre le milieu du XVIII siècle pour qu'un mouvement de colonisation se mette en branle dans la seigneurie. Bref, comme le souligne avec raison Valérie Nicolas:

Ce qui fait l'originalité de cette seigneurie, c'est qu'elle fut gérée, depuis 1697 jusqu'en 1854, par six générations de seigneurs provenant d'une même famille aux origines modestes qui l'obtint du noble Charles Denys de Vitré en échange d'une censive située sur l'lle d'Orléans, soit la famille Rioux, créant ce que l'on pourrait appeler, en empruntant les mots de Benoît Grenier, une dynastie seigneuriale ${ }^{11}$.

En raison de l'exceptionnelle longévité des Rioux à la tête de la seigneurie des Trois-Pistoles (la famille Rioux faisant en effet partie de la « dizaine de familles [qui] ont préservé leur fief de génération en génération, jusqu'à la fin, ou presque, du régime seigneurial $\left.{ }^{12}{ }^{\prime}\right)$, il semble tout à fait pertinent de faire porter notre réflexion sur l'implantation séculaire de cette famille dans sa seigneurie. Cette étude cherchera donc d'abord à discerner les causes de l'enracinement de la famille Rioux sur son fief. Par la suite, elle sera l'occasion d'analyser le déroulement de l'abolition du régime seigneurial à Trois-Pistoles. Finalement, elle déterminera la nature des traces mémorielles qui persistent à Trois-Pistoles ainsi que l'influence jouée par cette mémoire seigneuriale dans le rôle qu'ont occupé les descendants des seigneurs dans la communauté.

6. Laberge, Portraits de campagnes, p. 80.

7. Grenier, Brève histoire du régime seigneurial, p. 122.

8. Ibid., p. 130.

9. Valérie Nicolas, «La seigneurie de Trois-Pistoles à l'époque de la Nouvelle-France et au début du régime anglais: (1687-1784) », Mémoire de maîtrise (histoire), Université du Québec à Chicoutimi, (histoire) 2011, p. 49.

10. Ibid., p. 36.

11. Nicolas, «La seigneurie de Trois-Pistoles », p. 82.

12. Grenier, Brève histoire du régime seigneurial, p. 161. 


\section{De roturiers à seigneuris}

Alors que le régime français a vu 62 roturiers de statut modeste accéder à la propriété seigneuriale dans le gouvernement de Québec, le début du XIX siècle semble avoir été une période particulièrement difficile pour ces familles d'humble extraction puisque leur nombre diminue sensiblement durant toute cette période ${ }^{13}$. Cette décroissance continue est notamment perceptible au moment de l'abolition du régime seigneurial en 1854 puisqu'à ce moment les seigneurs descendants de familles modestes ne seront plus qu'une poignée à encore posséder le fief acquis par leurs ancêtres ${ }^{14}$. Avant de pousser plus loin notre recherche, la consultation de l'historiographie la plus récente disponible sur le sujet constitue l'occasion idéale afin de mieux saisir les principaux enjeux entrant en ligne de compte au moment d'étudier le maintien en place des familles seigneuriales d'origines modestes.

L'établissement de la famille Rioux dans la seigneurie de Trois-Pistoles en tant que maîtresse des lieux constitue certes « une incontestable ascension sociale ${ }^{15}$ », mais bien que les motifs exacts de la décision de Jean Rioux de s'implanter au Bas-Saint-Laurent soient impossibles à déterminer avec exactitude, tout porte à croire qu'il s'agit davantage d'un choix dicté par des impératifs pragmatiques que par un souci d'élévation du rang social. En effet, au moment où il échange sa censive de l'île d'Orléans contre le fief de Trois-Pistoles, le breton vise sûrement à assurer un avenir plus prometteur à ses fils. En effet, les terres de l'île d'Orléans ayant déjà toutes été concédées, ceux-ci ne peuvent espérer s'établir sur l'île et seront donc obligés de trouver un front pionnier où s'installer s'ils veulent obtenir une terre à cultiver. Comme le rappelle Benoît Grenier, «Les Rioux témoignent sans doute d'une attitude plus proche des familles d'habitants qui recherchaient dans la mobilité géographique des possibilités d'établissement pour leurs enfants ${ }^{16} »$. Cette intention originelle semble cruciale à prendre en compte afin de bien saisir le niveau d'enracinement de cette famille à TroisPistoles. En effet, les Rioux n'achètent pas le fief afin de l'ajouter à un ensemble seigneurial déjà bien garni comme le font certains seigneurs nobles ou fortunés, tel Charles Aubert de la Chesnaye. II se procurent plutôt la seigneurie en ayant comme objectif de faire de celle-ci un lieu de résidence permanent où ils pourront se fixer et établir les prochaines générations de la famille.

L'exemple des seigneurs Tremblay de la seigneurie des Éboulements est quant à lui révélateur du difficile maintien à la dignité seigneuriale chez les seigneurs d'origines modestes. En effet, alors que la seigneurie prend de plus en plus d'expansion au début du XIXe siècle,

13. Benoît Grenier, « «Écuyer, cultivateur»: des paysans devenus seigneurs en Nouvelle-France ou de l'ascension à l'exclusion du groupe seigneurial (17 - $19^{\mathrm{e}}$ siècles) ", dans Jean-Marc Moriceau et Philippe Madeline, dir., Les Petites Gens de la terre. Paysans, ouvriers et domestiques (Moyen Âge - XXle siècle), Caen, Presses de l'Université de Caen (Collection Bibliothèque du pôle rural \# 4), 2017, p. 95.

14. Ibid., p. 101.

15. Grenier, Brève histoire du régime seigneurial, p. 121.

16. Benoît Grenier, Seigneurs campagnards de la Nouvelle-France : Présence seigneuriale et sociabilité rurale dans la vallée du Saint-Laurent à l'époque préindustrielle, Rennes, Presses Universitaires de Rennes, 2007, p. 254. 
la famille Tremblay vend son fief à un médecin de Québec ${ }^{17}$. Mais pourquoi les Tremblay abandonnent-ils leur seigneurie alors que le développement de celle-ci commence à rendre le fief de plus en plus rentable? Selon Jean-Paul-Médéric Tremblay, c'est justement le développement croissant de la seigneurie qui rend le maintien des Tremblay difficile à la tête des Éboulements. En effet, les membres de la famille étant analphabètes, la gestion du fief devient « un fardeau administratif qui débord[e] la capacité et la compétence des seigneurs ${ }^{18}$ ", et ce d'autant plus que ce manque d'éducation a pour effet d'alourdir et rendre plus coûteuse l'administration seigneuriale en faisant de l'utilisation d'un notaire un incontournable afin de réaliser « toute action et transaction ${ }^{19}$ ».

Alors que le seigneur a été traditionnellement considéré par l'historiographie comme étant le « premier notable de la communauté20 ${ }^{20}$, il semble que ce rôle soit de plus en plus remis

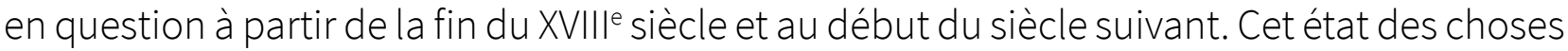
est le fruit d'une conjoncture où l'influence du seigneur d'humble extraction s'affaiblit au fur et à mesure que le pouvoir d'autres figures d'autorité émerge. L'historien Colin M. Coates affirme que le capitaine de milice et le curé constituent ces nouvelles figures d'autorité qui sont à même de concurrencer l'influence seigneuriale à partir de la fin du XVIII' siècle ${ }^{21}$.

L'hypothèse proposée dans cette étude se décline en trois temps se détaillant selon les parties suivantes du texte. Tout d'abord, l'origine modeste de la famille Rioux, l'adaptation de cette dernière aux réalités propres à la seigneurie de Trois-Pistoles, la présence des seigneurs Rioux sur leur fief et la densité du réseau familial seront les principaux éléments retenus pour défendre la thèse selon laquelle c'est l'enracinement de la famille dans son environnement, mais aussi auprès de la communauté, qui a permis à celle-ci de conserver aussi longtemps sa propriété seigneuriale. Par la suite, nous postulerons que le statut laïc des seigneurs de Trois-Pistoles a eu pour effet la multiplication des successions et donc la division de la possession seigneuriale entre les mains de plusieurs coseigneurs (rendant ainsi le processus d'abolition et son étude particulièrement complexe). Finalement, en ce qui concerne les traces et la mémoire découlant de l'enracinement seigneurial et familial des Rioux à Trois-Pistoles, nous soutiendrons l'idée selon laquelle l'ancienneté et la continuité de l'implantation à Trois-Pistoles a fait en sorte que la mémoire seigneuriale a été particulièrement vivace dans la région.

Afin de mener à bien cette recherche, plusieurs sources seront mobilisées. Tout d'abord, l'historiographie relativement abondante caractérisant la famille seigneuriale Rioux sera mise à profit. Ensuite, les rapports du Syndicat national du rachat des rentes seigneuriales

17. Grenier, «Écuyer, cultivateur», p. 100.

18. Jean-Paul-Médéric Tremblay, Être seigneur aux Éboulements, Baie-Saint-Paul, La Société d'histoire de Charlevoix, 1996, p. 134.

19. Ibid.

20. Grenier, Seigneurs campagnards, p. 212.

21. Colin M. Coates, Les transformations du paysage et de la société au Québec sous le régime seigneurial, Québec, Septentrion, 2003, p. 115. 
qui sont relatifs à la seigneurie de Trois-Pistoles permettront de mieux comprendre le déroulement du processus d'abolition du régime seigneurial dans ce fief. Finalement, les témoignages de Gaston Rioux (61 ans) et d'Anita Rioux (95 ans) qui ont été recueillis le 15 mai 2015 à Trois-Pistoles dans le cadre de la recherche sur les «Persistances seigneuriales au Québec » serviront à étayer notre réflexion relative aux traces et la mémoire du régime seigneurial à Trois-Pistoles. Ces témoignages seront particulièrement utiles en raison du fait que les individus interrogés sont des descendants directs de la famille seigneuriale, ce qui fait en sorte qu'ils portent davantage le bagage mémoriel de leur famille que d'autres témoins interrogés dans le cadre de la recherche.

\section{Enracinement et maintien de la dynastie seigneuriale des Rioux à Trois-Pistoles}

\section{Les Rioux : Une famille de basse extraction enracinée dans son milieu et sa commuinauité}

En débutant notre réflexion sur les causes de l'implantation séculaire de la famille seigneuriale Rioux dans la seigneurie de Trois-Pistoles, il convient en premier lieu de tâcher de comprendre comment cette famille a pu conserver son fief jusqu'à l'abolition de 1854 alors que tant d'autres familles d'origines semblables n'ont pas réussi, à commencer par leurs voisins, les Côté de l'Isle-Verte ${ }^{22}$. En arrivant dans la seigneurie de Trois-Pistoles au printemps 1697, Jean Rioux et sa famille deviennent à la fois les premiers seigneurs résidents et les premiers habitants de ce fief jusqu'alors inhabité. La localisation géographique excentrée de Trois-Pistoles de même que l'absence de voie d'accès autre que le fleuve Saint-Laurent ont également pour conséquence de laisser la famille Rioux sans censitaire jusqu'au milieu du XVIII ${ }^{e}$ siècle ${ }^{23}$. L'installation, le défrichement et la mise en valeur des premiers arpents de terre de cette seigneurie sont des tâches réalisées exclusivement par la famille Rioux durant plusieurs décennies. L'établissement progressif de colons sur le fief à partir de la seconde moitié du XVIII ${ }^{e}$ ne change pas non plus en profondeur le mode de vie de la famille seigneuriale puisque les rentes qui sont payées par les quelques censitaires présents à TroisPistoles sont loin d'être suffisantes afin d'assurer à elles seules la subsistance des Rioux. Ces derniers doivent donc continuer de cultiver leurs terres domaniales afin d'assurer leur survie, ce qui fait en sorte qu'ils mènent une vie plutôt semblable à celle de leurs censitaires $^{24}$. Alain Laberge note d'ailleurs à cet effet que les Rioux « semblent avoir exploité leur

22. Maude Flamand-Hubert, Louis Bertrand à l'Isle Verte, 1811-1871 : propriété foncière et exploitation des ressources, Québec, Presses de l'Université du Québec, 2012, 157 p.

23. Fortin et Lechasseur, Histoire du Bas-Saint-Laurent, p. 118. ; Benoît Grenier, « Jean Rioux : émigrant breton, seigneur canadien », Annales de Bretagne et des Pays de l'Ouest, tome 111, nº 3 (juin 2004), p. 86.

24. Grenier, « Jean Rioux : émigrant breton, seigneur canadien », p. 86. 
domaine comme une grosse censive dès le début de leur installation ${ }^{25} \gg$. Cet état des choses, qui fait en sorte que la famille seigneuriale peine à prime abord à se distinguer des autres résidents de Trois-Pistoles, appert se poursuivre jusqu'au moment de l'abolition en 1854. En effet, bien qu'à cette époque les Rioux possèdent leur fief depuis plus de 150 ans (ce laps de temps ayant probablement permis d'instaurer une certaine différenciation entre la famille seigneuriale et les autres familles pistoloises), Benoît Grenier souligne que «l'assimilation à la population paysanne locale » caractérisant la famille rend surprenante l'élection d’Éloi Rioux (dernier seigneur de Trois-Pistoles) au poste de maire de la municipalité ${ }^{26 .}$

De Jean à Éloi, les Rioux mènent donc une existence semblable à celle des autres résidents de leur fief. Cette quasi-absence de barrière socio-économique distinguant les Rioux et leurs censitaires contribue certainement au tissage de liens étroits entre la famille seigneuriale et les habitants, et ce, d'autant plus que la petite taille et l'isolement du fief favorisent les rapprochements entre les gens présents sur celui-ci ${ }^{27}$. Benoît Grenier confirme d'ailleurs que cette réalité est propre aux seigneuries possédées par des seigneurs de basse extraction lorsqu'il explique que règle générale, « les familles seigneuriales plus modestes vont entretenir des rapports constants avec la paysannerie ${ }^{28} »$. Ces rapports de proximité qui se nouent entre les Rioux et les résidents de la seigneurie sont cruciaux dans la compréhension du niveau d'ancrage de la famille seigneuriale sur son fief, car comme le rappelle Jacques Mathieu «l'enracinement paraît très fortement lié à une sorte d'intégration sociale ${ }^{29}$ ».

Mais l'enracinement des Riouxà Trois-Pistoles provient aussi fort probablement du lien émotif développé au fil du temps envers le fief et le titre hérité des ancêtres. En effet, même s'ils savent que leur seigneurie lointaine ne peut « guère leur conférer le prestige habituellement reconnu aux seigneurs ${ }^{30}$ », les Rioux sont néanmoins " assurément conscients de la «dignité» de ces terres » qui sont les leurs ${ }^{31}$. Cette pleine compréhension du statut privilégié relié à la possession d'un fief au sein d'une société où le régime seigneurial introduit «la supériorité sociale d'un individu [le seigneur] sur d'autres [les censitaires] » entraîne donc sûrement un désir de perpétuation de ce rang social qui donne à la famille une distinction avantageuse par rapport aux autres habitants de Trois-Pistoles ${ }^{32}$. À cet égard, l'enracinement de la lignée seigneuriale peut donc également être considéré comme une conséquence du désir des générations successives de seigneurs Rioux de préserver le «vieux bien » acquis par les aïeux

25. Alain Laberge, «Propriété et développement des seigneuries du Bas-Saint-Laurent (1670-1790) », dans Serge Courville et Jacques Mathieu, dir., « Le peuplement colonisateur aux XVIIe et XVIIIe siècles », Cahiers du Célat, n 8 (1987), p. 225.

26. Grenier, « Jean Rioux : émigrant breton, seigneur canadien », p. 86. ; Grenier, Seigneurs campagnards, p. 229.

27. Fortin et Lechasseur, Histoire du Bas-Saint-Laurent, p. 137.

28. Grenier, Brève histoire du régime seigneurial, p. 170.

29. Jacques Mathieu, La Nouvelle-France : les Français en Amérique du Nord, XVIIe -XVIIIe siècle, Québec, Presses de l'Université Laval, 2001 (1991), p. 93.

30. Grenier, «Écuyer, cultivateur», p. 96.

31. Ibid.

32. Grenier, Brève histoire du régime seigneurial, p. 33. 
et pour lequel un attachement identitaire s'est développé au fil du temps et des efforts investis afin de transformer une terre en «bois debout » en un fief cultivé et habité de manière permanente ${ }^{33}$.

\section{Le réseau familial, un gage d'ancrage dans la communauté?}

À Trois-Pistoles, la famille Rioux est omniprésente. Cette réalité singulière est illustrée par Benoît Grenier lorsqu'il affirme que l'« on aura bien du mal à trouver quelqu'un qui ne soit pas un descendant de Jean Rioux » dans ce fief du Bas-Saint-Laurent ${ }^{34}$. Selon toute vraisemblance, la densité du réseau familial dans lequel évolue la lignée seigneuriale est un élément central expliquant le degré d'enracinement de cette dernière. En effet, durant environ cinquante ans, la seigneurie de Trois-Pistoles se développe avec comme seul apport démographique extérieur les hommes et les femmes venant dans le fief afin d'épouser des Rioux. L'administration du fief pendant cette période pionnière s'apparente ainsi à « une logique strictement familiale » dans laquelle « les relations seigneurs/censitaires ne sont certainement pas celles que l'historiographie marxiste a dépeinte ${ }^{35}$ ». Dans une société où « la famille représentait le principal moyen d'expression de son existence », il est donc tout à fait compréhensible que les membres de la famille Rioux cherchent à rester le plus près des leurs, ce qui passe alors nécessairement par l'enracinement au sein du fief familial ${ }^{36}$. Cependant, une fois que la période pionnière est dépassée et que la seigneurie se retrouve habitée par un nombre croissant de censitaires venus de l'extérieur, l'importance accordée au réseau familial des Rioux dans l'enracinement de ces derniers dans leur seigneurie n'est-elle pas à récuser? En fait, la présence des Rioux dans le fief demeure non négligeable puisqu'en plus de la prolifération continuelle des branches collatérales de la famille, la lignée seigneuriale principale connaît un niveau de reproduction particulièrement important (les femmes des six seigneurs Rioux se relayant à la tête du fief ayant en moyenne 10 enfants chacune ${ }^{37}$ ). Cette réalité nous pousse à affirmer que le réseau familial occupe une place structurante dans la fixation des Rioux à Trois-Pistoles, et ce, à travers les différentes époques.

Mais que signifie concrètement la présence d'une forte proportion de Rioux dans la seigneurie? En fait, tout porte à croire que la présence importante de membres de leur propre famille au sein de leur fief constitue pour les Rioux un avantage crucial. En effet, dans le Québec préindustriel, « la famille est [...] l'institution de base » puisqu'elle constitue « le cadre à l'intérieur duquel les gens ont mis au point leurs stratégies économiques et sociales et ont tenté d'assurer la stabilité d'une génération à l'autre ${ }^{38} »$. Le fait qu'une partie substantielle des habitants de la seigneurie fasse partie intégrante de ce « cadre » solidaire envers

33. Grenier, Seigneurs campagnards, p. 73.

34. Grenier, «Écuyer, cultivateur», p. 101.

35. Ibid., p. 98.

36. Coates, Les transformations, p. 73.

37. Grenier, Seigneurs campagnards, p. 68.

38. Coates, Les transformations, p. 79. 
la dynastie seigneuriale constitue donc un atout appréciable pour une lignée seigneuriale de basse extraction pour qui la conservation de la propriété du fief est loin d'être assurée. Il convient toutefois de nuancer quelques peu les explications fournies précédemment en soulignant que les nombreuses branches de la famille Rioux, même si elles partent toutes d'un ancêtre commun, finissent par se distancier à mesure que le temps passe et que le lien commun que constitue l'aïeul collectif s'estompe progressivement des mémoires.

À la veille de l'abolition de 1854, il semble donc illusoire de s'imaginer que l'ensemble des Rioux de Trois-Pistoles s'insère dans une relation de stricte proximité avec la lignée seigneuriale principale. Cette nuance ne remet cependant pas en cause les fondements de cette démonstration puisque nous postulons l'idée que malgré l'écart croissant qui se creuse entre certaines branches de la famille au gré des générations, la lignée des seigneurs principaux a toujours pu miser sur une parenté nombreuse sur laquelle il était possible pour elle de compter.

\section{Le maintien des Rioux à la tête du fief de Trois-Pistoles}

Le maintien des familles seigneuriales d'origines modestes à la tête de leur fief devient particulièrement difficile à partir du début du XIXe siècle. Celles-ci doivent en effet composer avec plusieurs facteurs (analphabétisme, émiettement du fief, déficience de la reproduction familiale, concurrence d'élites locales, etc.) qui rendent la conservation de la dignité seigneuriale plus difficile et parfois même intenable. Examinons maintenant ces différents éléments influençant le maintien seigneurial des seigneurs de basse extraction afin de déterminer comment les Rioux se situent par rapport à ceux-ci. On pourra ainsi mieux comprendre pourquoi la famille seigneuriale de Trois-Pistoles a réussi là où la majorité de ses semblables ont échoué. Tout d'abord, le niveau d'alphabétisation est déterminant dans la capacité d'une lignée seigneuriale à se maintenir en place. En effet, la hausse constante de la population coloniale fait en sorte que les seigneuries autrefois peu peuplées se retrouvent habitées par un nombre croissant de censitaires, ce qui rend inévitablement plus ardu la gestion de «l'ensemble des opérations liées à la perception des droits seigneuriaux, mais aussi à l'organisation de l'espace ${ }^{39} »$. Pour pouvoir mener à bien la gestion de leurs fiefs de plus en plus populeux, les seigneurs se doivent donc de savoir lire et écrire, car le « fardeau administratif » accompagnant de plus en plus la possession d'une seigneurie rend presque obligatoire l'acquisition par la caste seigneuriale d'un « niveau éducationnel minimal ${ }^{40}$ ».

À défaut de se doter d'une éducation, les seigneurs peuvent toujours se tourner vers les services de notaires ou de lettrés étant en mesure de réaliser pour eux la partie « administrative » des fonctions dévolues à un seigneur, mais l'emploi d'un tel intermédiaire représente une dépense que les seigneurs d'humble extraction ne peuvent pas nécessairement se permettre, comme en témoigne la vente de la seigneurie de l'Islet-de-Bonsecours à la fin du XVIII siècle par une famille seigneuriale d'origines modestes (les Bélanger) étant aux

39. Grenier, Seigneurs campagnards, p. 90.

40. Tremblay, Être seigneur aux Éboulements, p. 134. ; Grenier, Seigneurs campagnards, p. 90-91. 
prises avec plusieurs difficultés, dont un problème financier d'une grande importance ${ }^{41}$. Au XIXe siècle, les familles seigneuriales de basse extraction ne réussissant pas à s'instruire sont donc pour la plupart contraintes de remettre leur fief « entre les mains de propriétaires mieux outillés pour gérer une seigneurie ${ }^{42} »$.

Mais quelle est la situation au sein de la dynastie seigneuriale des Rioux de Trois-Pistoles? De 1709 à 1784, les deuxième et troisième seigneurs Rioux savent écrire, et ce malgré l'absence de cette capacité chez le couple formé de Jean Rioux et de Catherine Leblond ${ }^{43}$. Cependant, les quatrième et cinquième seigneurs Rioux (ceux-ci dirigent le fief entre 1784 et 1829) ne peuvent même pas se targuer de savoir écrire leur nom ${ }^{44}$. Cette perte d'instruction au sein de la lignée seigneuriale, qui est sans doute reliée à la diminution progressive de l'alphabétisation dans la colonie durant le XVIII' siècle, est cependant freinée par « l'amélioration du système scolaire au cours du XIXe siècle » puisqu'Éloi Rioux, le sixième et dernier seigneur de Trois-Pistoles, sait manifestement écrire ${ }^{45}$.

Pendant la période charnière de la fin du XVIII e siècle et du début du XIX siècle, où tant de seigneurs de basse extraction perdent leur fief, les seigneurs Rioux de Trois-Pistoles ont donc été analphabètes. De plus, ceux-ci n’ont généralement pas pu compter sur leurs frères et sœurs afin de combler cette lacune puisque, comme l'indique Benoît Grenier, « certaines familles ou générations sont complètement analphabètes » comme lorsqu'au commencement du XIXe siècle " aucun des six enfants du seigneur Étienne Rioux II [...] ne furent en mesure d'apposer leur signature à leur contrat de mariage ${ }^{46} »$. L'arrivée d'un seigneur lettré (Éloi Rioux) aux commandes du fief en 1829 et l'installation d'une école dans la seigneurie la même année semblent apporter une solution durable aux difficultés d'alphabétisation des seigneurs de Trois-Pistoles, mais ces solutions, qui arrivent à peine 25 ans avant l'abolition du régime seigneurial, sont tardives et n'expliquent pas comment les Rioux ont pu surpasser les problèmes engendrés par leur analphabétisme à un moment où la connaissance de la lecture et de l'écriture se révèle justement plus importante que jamais auparavant ${ }^{47}$.

La dynastie seigneuriale aurait-elle pu compenser son déficit d'instruction en menant à bien une reproduction familiale efficace? La réponse à cette question revêt une importance cruciale puisque la réussite ou l'échec d'une reproduction familiale «à l'identique » est un autre élément qui entre en compte dans la réussite ou l'échec du maintien du statut seigneurial chez les seigneurs d'origines modestes. En effet, selon Benoît Grenier, les seigneurs qui dé-

41. Grenier, Seigneurs campagnards, p. 90-91; Raymond Bélanger, François Bellenger: Seigneur de l'Islet-de-Bonsecours, Québec, Les Presses de l'Université Laval, 2010, p. 331.

42. Grenier, Brève histoire du régime seigneurial, p. 154.

43. Nicolas, «La seigneurie de Trois-Pistoles », p. 51.

44. Grenier, Seigneurs campagnards, p. 93.

45. Michel Verrette, «L’alphabétisation au Québec, 1660-1900 », Thèse de doctorat, Université Laval, (histoire) 1989, p. 148 ; Mathieu, La Nouvelle-France, p. 210. ; Grenier, Seigneurs campagnards, p. 93-94.

46. Grenier, Seigneurs campagnards, p. 93.

47. Ibid., p. 94. 
sirent perdurer à la tête de leur fief se doivent d'« acquérir et [de] maintenir une position socioculturelle légitimant la nature de la propriété possédée ${ }^{48} »$. Or, l'obtention d'une telle " position socioculturelle » passe notamment par une reproduction familiale où les enfants du seigneur parviennent à conserver un certain rang social, car si la progéniture seigneuriale ne peut faire mieux que de s'établir sur des censives comme les autres enfants de la seigneurie, la dynastie seigneuriale de basse extraction est susceptible de se voir entraîner dans une spirale descendante la ramenant à son statut paysan d'origine ${ }^{49}$. Mais qu'en est-il de la situation chez les seigneurs Rioux? Tout d'abord, les alliances matrimoniales que contractent les premières générations ont tendance à se caractériser par une certaine homogamie puisque les conjoints sélectionnés proviennent en bonne partie des familles seigneuriales Côté et Lepage de l'Isle-Verte et Rimouski50. Cependant, un tournant s'opère à la toute fin du XVIII siècle quand le peuplement du fief rend possible pour les enfants des seigneurs Rioux de se trouver des conjoints au sein même de la seigneurie ${ }^{51}$. À partir de ce moment, les alliances tissées par la famille seigneuriale le sont généralement sous le signe de l'hypogamie, car les héritiers Rioux se marient alors règle générale avec d'humbles résidents de Trois-Pistoles ${ }^{52}$. Bref, au moment même où le maintien en place des familles seigneuriales de basse extraction devient de plus en plus difficile, les Rioux se mettent à conclure des unions qui s'opposent au principe selon lequel « le mariage vise habituellement à s'allier à quelqu'un de son groupe social, ou mieux encore, appartenant à une catégorie sociale plus élevée ${ }^{53}$ ».

Ce déclassement social, qui ne favorise pas chez les Rioux « l'affermissement d'une position sociale dominante », ne semble cependant pas avoir eu un impact aussi négatif que chez d'autres seigneurs de condition semblable. En effet, selon Raymond Bélanger, c'est l'échec d'une reproduction « à l'identique » qui peut être considéré comme le facteur principal expliquant la perte du fief de l'Islet-de-Bonsecours par la famille Bélanger ${ }^{54}$. Comment expliquer la persistance de la lignée seigneuriale de Trois-Pistoles face à ces difficultés qui se révèlent pourtant fatales à d'autres dynasties seigneuriales de la vallée du Saint-Laurent? La réponse se trouve peut-être dans le fait qu'à défaut de conserver une position sociale dominante, les Rioux vont développer " des rapports constants avec la paysannerie » afin de compenser leur absence de suprématie sociale par un enracinement au sein de la masse populaire ${ }^{55}$. Maintenant, que le niveau d'alphabétisation et la nature de la reproduction familiale ont été examinés, il convient d'explorer les autres facteurs influençant la réussite ou l'échec du maintien en place des familles seigneuriales de modeste extraction.

48. Ibid., p. 252.

49. Ibid., p. $75-81$

50. Ibid., p. 124.

51. Ibid.

52. Grenier, Brève histoire du régime seigneurial, p. 170.

53. Grenier, Seigneurs campagnards, p. 122.

54. Bélanger, François Bellenger: Seigneur de l'Islet-de-Bonsecours, p. 331.

55. Grenier, Brève histoire du régime seigneurial, p. 170. 
La capacité ou non d'une famille seigneuriale à éviter l'émiettement de son fief constitue l'un des autres facteurs entrant en ligne de compte dans l'échec ou la réussite du maintien seigneurial. En effet, même si la coutume de Paris permet plus facilement aux seigneurs qu'aux censitaires d'éviter le morcellement de la terre, l'émiettement des seigneuries subséquentes aux partages successoraux ne reste pas moins une caractéristique des fiefs laïcs (les seigneuries appartenant à des communautés religieuses étant logiquement exemptées des divisions inhérentes aux successions familiales $\left.{ }^{56}\right)$. Selon Benoît Grenier, le fractionnement de la seigneurie «entre les cohéritiers et les partages successifs furent en partie responsables de la perte de la propriété, de par la présence d'une multitude de petits coseigneur ${ }^{57}$ ». Ces coseigneurs, qui partent à chaque génération avec une partie des terres du fief, affaiblissent donc inlassablement le patrimoine revenant au fils aîné, ce dernier ne pouvant alors plus transmettre à ses propres descendants un fief aussi bien doté que celui qu'il a reçu. C'est donc pour empêcher que le seigneur principal du fief ne se retrouve dans une position économique intenable suite à un morcellement trop prononcé de son héritage que « la volonté d'éviter le fractionnement de la seigneurie est fort probable parmi les familles seigneuriale ${ }^{58}$ ». Mais comment la dynastie des Rioux a-t-elle fait face à ce défi se posant à tous les seigneurs laïcs? Alain Laberge affirme que les seigneuries du Bas-Saint-Laurent se sont caractérisées par « un morcellement parfois fort complexe », mais il rajoute du même souffle que « cet émiettement ne semble pas avoir eu d'incidences directes sur les stratégies seigneuriale ${ }^{59}$ ». Cette description concorde effectivement avec la situation en vigueur à TroisPistoles puisque le morcellement du fief commence dès le début du XVIII' siècle au moment où les fils de Jean Rioux se partagent la seigneurie ${ }^{60}$. Cet émiettement s'accélère par la suite de façon constante en raison de la progéniture particulièrement nombreuse engendrée par les seigneurs Rioux qui se relaient à la tête du fief. Cependant, comme le mentionne Alain Laberge, la division de la seigneurie ne semble pas avoir eu des conséquences trop importantes pour les Rioux puisque malgré un émiettement particulièrement important (en 1863 les commissaires qui rédigent le cadastre abrégé notent la présence de 53 co-propriétaires), ils n'ont pas perdu la propriété du fief ancestral ${ }^{61}$.

Cette « résistance » à un morcellement qui a, dans plusieurs cas, obligé des dynasties seigneuriales à se départir de leur fief ancestral ne constitue cependant pas un cas unique. En effet, les seigneurs Chicoine de la seigneurie de Bellevue, qui réussissent tout comme les Rioux à conserver leur seigneurie jusqu'à l'abolition de 1854, ont également connu un émiettement important de leur fief (le commissaire chargé d'évaluer la seigneurie dénom-

56. Grenier, Seigneurs campagnards, p. 76. ; Grenier, Brève histoire du régime seigneurial, p. 124-125.

57. Grenier, Seigneurs campagnards, p. 87.

58. Ibid., p. 73-88.

59. Laberge, «Propriété et développement», p. 219.

60. Nicolas, «La seigneurie de Trois-Pistoles », p. 54-56.

61. Cadastres abrégés des seigneuries de Québec, Montréal, Trois-Rivières et de la Couronne, Québec, Derbishire et Desbarats, 1864, 7 volumes : seigneurie de Trois-Pistoles. 
brant ainsi 15 coseigneurs dans le cadastre abrégé qu'il publie en $1857^{62}$ ). Bref, le morcellement des fiefs, qui représente un problème majeur pour les familles seigneuriales laïques désirant conserver leur seigneurie, n’a pas épargné la lignée des Rioux et a même été d’une importance singulière à Trois-Pistoles. Cependant, l'exemple fourni par la famille Chicoine témoigne du fait que ce facteur ne représente pas un danger insurmontable à la pérennisation d'un fief.

Maintenant que nous avons constaté que la dynastie seigneuriale des Rioux s'est caractérisée par un analphabétisme partiel, une reproduction familiale généralement peu « réussie » et un fief en émiettement croissant, il est temps de se pencher sur la capacité de cette famille à s'insérer au sein de la notabilité locale qui est en pleine émergence à compter de la fin du XVIII siècle et qui remet par le fait même en question la prédominance seigneuriale. En effet, cette période voit la domination du seigneur devenir de moins en moins structurante puisque:

La composition de l'élite a changé avec le temps. Engagés à fond dans le règlement des questions locales sous le régime français, les seigneurs avaient pris leurs distances au début du XIXe siècle. Entre-temps, le clergé local avait accru son influence, comme les capitaines de milice d'ailleurs. Avec le temps, le phénomène de différenciation économique dans les campagnes avait entrainé la création d'une petite-bourgeoisie de marchands et d'agriculteurs de grande envergure63.

En plus du curé, du capitaine de milice et de la petite-bourgeoisie locale, un autre acteur social influent est identifié par Donald Fyson lorsque celui-ci affirme que «la plupart des juges de paix sont des personnes qui jouissent au moins d'une certaine notoriété locale ${ }^{64}$ ». Mais en quoi la concurrence qu'exercent ces différents individus face au pouvoir seigneurial constitue-t-elle une menace vis-à-vis du maintien en place des familles seigneuriales d'humble extraction? En fait, le danger pour les lignées seigneuriales d'origines modestes provient du fait que celles-ci ont généralement fait de l'enracinement dans la communauté locale leur point d'ancrage principal afin de demeurer en possession de leur fief. Or, en perdant son rôle de "premier notable de la communauté », le seigneur en vient aussi à être moins sollicité afin d'intervenir dans les affaires de la localité, ce qui fait en sorte qu'il se retrouve peu à peu « moins intégré à la collectivité65 ». C'est donc l'enracinement de la dynastie seigneuriale au sein de son propre fief qui est remis en question lorsque la nouvelle élite qui se forme dans le monde seigneurial durant les années qui suivent la Conquête entreprend une ascension sociale souvent préjudiciable à l'influence seigneuriale. Certains seigneurs, incapables de faire face à cette concurrence, se départissent de leur fief ou se retrouvent

62. André Chicoine, La seigneurie de Bellevue: Patrimoine des familles Chicoine et Boisseau à Verchères, Longueuil, André Chicoine, 2015, p. 158.

63. Coates, Les transformations, p. 95-96.

64. Donald Fyson, Magistrats, police et société : La justice criminelle ordinaire au Québec et au Bas-Canada (1764-1837), Montréal, Éditions Hurtubise, 2010, p. 209.

65. Grenier, Seigneurs campagnards, p. 212 ; Coates, Les transformations, p. 121. 
complètement déclassés socialement (ce sera notamment le cas des Côté de L'Isle-Verte ${ }^{66}$ ). D’autres, conscients de l'effritement du pouvoir seigneurial traditionnel, vont tisser des liens avec la notabilité émergente afin de l'intégrer ou de s'y associer ${ }^{67}$.

À Trois-Pistoles, à partir du moment où la population atteint un certain seuil (le cap des 1000 habitants est franchi entre 1790 et 1825), la famille seigneuriale ne peut déjà plus être considérée comme possédant une notabilité incontestable au sein de la seigneurie ${ }^{68}$. En effet, la prestigieuse fonction de capitaine de milice, qui fut l'apanage des seigneurs Rioux pendant de longues années, échappe au monopole de la famille seigneuriale " principale » de sorte qu'au début du XIXe siècle c'est Paul Rioux (un membre d'une branche collatérale de la lignée seigneuriale principale) qui occupe cette position influente ${ }^{69}$. Un autre exemple de cette influence montante de la nouvelle élite locale s'incarne aussi à travers le marchand Philippe Renouf qui constitue une figure centrale de la notabilité pistoloise au cours du XIXe siècle. Cette position privilégiée, qui lui permet de concurrencer directement les seigneurs Rioux, favorise aussi l'appropriation de prérogatives autrefois réservées à ces derniers, tel le rôle de «donateur du terrain pour la construction d’une nouvelle église ${ }^{70}$ ».

La croissance de cette notabilité pistoloise remet donc en question l'unicité du pouvoir seigneurial, mais ne semble pas pour autant se traduire par un déclassement complet des seigneurs Rioux. En effet, ceux-ci voient plutôt leur rôle de premiers notables se transformer en une position de notables parmi d'autres. Ce positionnement au sein de la notabilité locale est cependant fragile, car comme nous l'avons observé précédemment l'analphabétisme, la reproduction familiale et l'émiettement du fief sont autant d'éléments qui entraînent la position sociale des Rioux à la baisse. Mais il n'empêche que l'appartenance des Rioux à la classe des notables de Trois-Pistoles est décelable, et ce grâce à plusieurs éléments qui peuvent apparaitre à première vue anecdotiques. D'une part, bien que ce ne soit pas la norme, la famille seigneuriale réalise un certain nombre d'alliances matrimoniales avec la notabilité pistoloise, comme en témoigne le mariage célébré en 1834 entre Marie-Angélique Rioux (la fille du seigneur Joseph Rioux) et le notaire Pierre Fournier ${ }^{71}$. D'autre part, il est intéressant de constater que durant la « chicane des églises » ayant divisé la seigneurie de 1827 à 1853, la famille seigneuriale a rejoint le camp « d'en bas » (c'est-à-dire celui formé « des personnes les plus marquantes, les plus anciennes, des pères [...] de la paroisse, puis des plus riches puisque plus anciennement établis ») plutôt que le camp « d'en haut » (c'est-à-dire celui composé de la majeure partie des résidents du fief). Ce ralliement au «camp » constitué de l'élite locale témoigne que malgré leur modestie et leur liens étroits avec la paysannerie, les seigneurs Rioux n'en sont pas moins membres d'une certaine élite à laquelle l'adhésion

66. Grenier, Brève histoire du régime seigneurial, p. 160.

67. Grenier, Seigneurs campagnards, p. 246.

68. Fortin et Lechasseur, Histoire du Bas-Saint-Laurent, p. 137.

69. Grenier, Seigneurs campagnards, p. 350.

70. Ibid., p. 253.

71. Ibid., p. 247. 
n’est pas seulement assurée par la richesse, mais aussi par le prestige et la notoriété72.

En conclusion, cette première partie a permis d'illustrer à quel point malgré ses origines modestes la famille seigneuriale Rioux a su mettre à profit son enracinement séculaire ainsi que son large réseau familial afin de s'implanter durablement à la tête de son fief. Le début du XIXe, moment où tant de familles seigneuriales de basse extraction perdent leur seigneurie, est cependant porteur de changement pour cette famille seigneuriale qui voit son règne sans partage sur la seigneurie être relégué aux oubliettes suite à l'émergence d'une notabilité locale gagnant de plus en plus d'influence à Trois-Pistoles. Face à la concurrence qu'entraîne inévitablement l'essor de cette nouvelle élite locale, les seigneurs Rioux ne semblent, à première vue, posséder aucun atout leur permettant de conserver la mainmise sur leur fief puisqu'ils sont complètement analphabètes durant deux générations (c'est-à-dire de 1784 à 1829), qu'ils contractent des mariages étant généralement caractérisés par l'hypogamie et qu'ils voient leur seigneurie s'émietter sans arrêt au gré des partages successoraux successifs. Cependant, leur position sociale prestigieuse héritée de leur statut de seigneurs des lieux et de pionniers du fief semble leur permettre de tisser des liens avec les notables locaux et donc de s'insérer au sein des réseaux de pouvoir qui se forment dans la région au cours du XIXe siècle. C'est donc grâce à un enracinement local incontestable, un réseau familial bien développé et une position sociale leur donnant une influence tant au sein de la notabilité que de la paysannerie que les seigneurs Rioux ont réussi à pérenniser leur dynastie seigneuriale pourtant bien modeste.

\section{L’abolition du régime seigneurial et ses conséquences à Trois-Pistoles}

\section{Une abolition seigneuriale progressive}

Au moment où le régime seigneurial est aboli en 1854, celui-ci impose sa marque à la société et au territoire québécois depuis 230 ans. En effet, lorsque la France entreprend la colonisation de sa colonie nord-américaine au début du XVII ${ }^{e}$ siècle, elle décide naturellement d'y implanter le même système que celui ayant cours chez elle ${ }^{73}$. La seigneurie, qui représente « à la fois un espace sur lequel s'exercent les droits d'un seigneur et une forme de propriété foncière », devient donc ainsi l'institution française du Nouveau Monde ayant « précédé tout le reste ${ }^{74} »$. Le régime seigneurial se voit cependant de plus en plus critiqué sous le régime britannique ${ }^{75}$. En effet, comme le résume Benoît Grenier:

72. Ibid., p. 349-350.

73. Fernand Ouellet, «Propriété seigneuriale et groupes sociaux dans la vallée du Saint-Laurent (1663- 1840) », Revue de l'Université d'Ottawa, vol. 47, n 1, 1977, p. 183-195.

74. Louise Dechêne, Habitants et marchands de Montréal au XVII e siècle, Montréal, Boréal, 1988, p. 241.

75. Grenier, Brève histoire du régime seigneurial, p. 140-141. 
Par les restrictions qu'il impose et par les monopoles qu'il implique, le régime seigneurial constitue, à l'heure de l'industrialisation, une entrave évidente à l'esprit d'entreprise, à la libre propriété et à la liberté contractuelle, en somme au développement du capitalisme ${ }^{76}$.

Dès 1801, l'abolition de la tenure seigneuriale est donc suggérée par une commission d'enquête, mais ce n'est qu'en 1854 que l'abolition du régime est finalement adoptée en chambre. Les députés siégeant au parlement du Canada-Uni optent alors pour « la commutation obligatoire des censives en tenure franche contre une compensation financière aux seigneurs », ce dernier élément étant lourd de conséquence pour la suite des choses puisqu'en plus de conserver leur domaine les seigneurs seront dédommagés financièrement pour « la perte de la propriété éminente sur les censives ${ }^{77}{ }^{»}$.

\section{L'abolition à Trois-Pistoles : regard sur une seigneurie morcelée}

Mais comment l'abolition progressive du régime seigneurial s'est-elle déroulée à TroisPistoles? Afin de répondre à cette question, le cadastre abrégé réalisé suite à l'adoption de l'Acte seigneurial de 1854 et les archives du Syndicat national du rachat des rentes seigneuriales seront notamment mis à contribution. En 1858, le cadastre de la seigneurie est clos après que le commissaire Siméon Lelièvre ait réalisé un examen approfondi de la propriété de chaque coseigneur présent dans le fief ${ }^{78}$. Le travail du commissaire permet tout d'abord d'exposer l'état avancé de morcellement de la seigneurie. En effet, Siméon Lelièvre souligne que « la seigneurie en question est subdivisée entre cinquante-trois copropriétaires, vingtquatre desquels possèdent des parts sur lesquelles sont établis des Censitaires, et vingtneuf possèdent leur terre en fief et comme copropriétaires de la dite Seigneurie ${ }^{79}$ ». Le cadastre étant rédigé afin de compenser les seigneurs pour la perte de la propriété éminente sur des censives, le travail réalisé par Lelièvre en 1858 ne porte donc que sur les vingt-quatre copropriétaires ayant des censitaires installés sur leurs terres. Parmi ces derniers, certains attirent davantage notre attention que d'autres.

La première partie du fief, qui correspond à la propriété du capitaine Jean-Baptiste Rioux, représente par exemple la propriété la plus lucrative du fief $(8358,23 \$)$ puisqu'elle compte le nombre le plus élevé de censitaires (c'est-à-dire 171 habitants) ainsi qu'un vaste domaine. La troisième partie de la seigneurie consiste quant à elle en la propriété du seigneur principal des lieux, c'est-à-dire le « seigneur primitif » Éloi Rioux ${ }^{80}$. Le seigneur Rioux compte 141 censitaires dans sa partie du fief, et contrairement aux autres coseigneurs de Trois-Pistoles, il possède les terres non concédées de la seigneurie. La valeur de sa part du fief s'élève donc à 7 830,57\$, ce qui en fait le deuxième propriétaire le plus riche de la seigneurie de

76. Ibid., p. 192.

77. Ibid., p. 203-204.

78. Cadastres abrégés de la seigneurie de Trois-Pistoles.

79. Ibid.

80. Ibid. 
Trois-Pistoles. La cinquième partie du fief est celle appartenant au coseigneur Nazaire Têtu. Ce commerçant, cousin du riche marchand Félix Têtu, ne possède aucun domaine et ne déclare que deux censitaires, ce qui fait en sorte que sa parcelle seigneuriale ne vaut que $175,34 \${ }^{81}$. La dix-huitième partie du fief est quant à elle particulière puisqu'elle a une valeur totale de 0,33\$. Son propriétaire, Raphaël Plourde, ne possède en effet aucun domaine et seulement un censitaire est établi dans sa part de la seigneurie, expliquant ainsi ce faible montant. Pour finir, le marchand, commissaire scolaire et maire de Trois-Pistoles Philippe Renouf détient une part de la seigneurie où sont établis trois censitaires ${ }^{82}$. C'est donc grâce à son imposant domaine que Renouf voit la valeur de sa partie de la seigneurie s'élever à l'appréciable somme de $2956 \$ 33$. Bref, le cadastre abrégé de 1858 permet de constater que malgré le morcellement caractérisant le fief de Trois-Pistoles, la famille Rioux y occupe toujours une place non négligeable puisque dix des vingt-quatre parts de la seigneurie appartiennent toujours à des descendants de Jean Rioux et que ces dix parts comptent parmi les plus importantes du fief.

Que nous apprend donc le contenu du cadastre abrégé de 1858 sur l'état de la seigneurie de Trois-Pistoles au moment de l'abolition? D'abord, que l'émiettement du fief a des conséquences significatives sur la propriété seigneuriale puisque le seigneur principal Éloi Rioux se retrouve à être en possession d'une part d'une moindre valeur que celle de Jean-Baptiste Rioux. Ce dernier est l'héritier de l'influent capitaine de milice Paul Rioux qui est présenté par le curé en poste à Trois-Pistoles en 1845 comme ayant été de son vivant le « coseigneur le plus riche et le plus grand propriétaire de cette paroisse ${ }^{84} »$. Cet état des choses permet de constater à quel point, en ce milieu du XIX siècle, le seigneur principal est fortement concurrencé par d'autres coseigneurs et ce, non seulement au point de vue économique, mais aussi au point de vue social. En effet, en plus de percevoir plus de rentes seigneuriales qu'Éloi Rioux, Jean-Baptiste Rioux peut se targuer d'avoir davantage de censitaires sous son influence que le seigneur primitif. L'influence qu'exercent les nombreux coseigneurs au sein du fief se fait aussi sentir au niveau du moulin seigneurial puisque celui-ci n'est pas la propriété exclusive d’Éloi Rioux. En effet, en plus du seigneur principal, quatre coseigneurs possèdent des parts dans cet immeuble occupant une place de choix dans la sociabilité et l'économie locale ${ }^{85}$. Ce partage d'un bâtiment incarnant le monopole seigneurial (les censitaires ayant l'obligation de faire moudre leurs grains dans le moulin seigneurial) témoigne donc de l'influence déclinante de la lignée principale et de la montée en puissance de coseigneurs qui sont à même de rivaliser avec la famille principale en ce qui concerne le contrôle des institutions seigneuriales ${ }^{86}$.

81. Emmanuel Rioux, dir., Histoire de Trois-Pistoles : 1697 - 1997, Trois-Pistoles, Centre d'édition des Basques, 1997, p. 438.

82. Ibid., p. 431.

83. Cadastres abrégés de la seigneurie de Trois-Pistoles.

84. Rioux, dir., Histoire de Trois-Pistoles, p. 438-439.

85. Cadastres abrégés de la seigneurie de Trois-Pistoles.

86. Laberge, Portraits de campagnes, p. 108. 


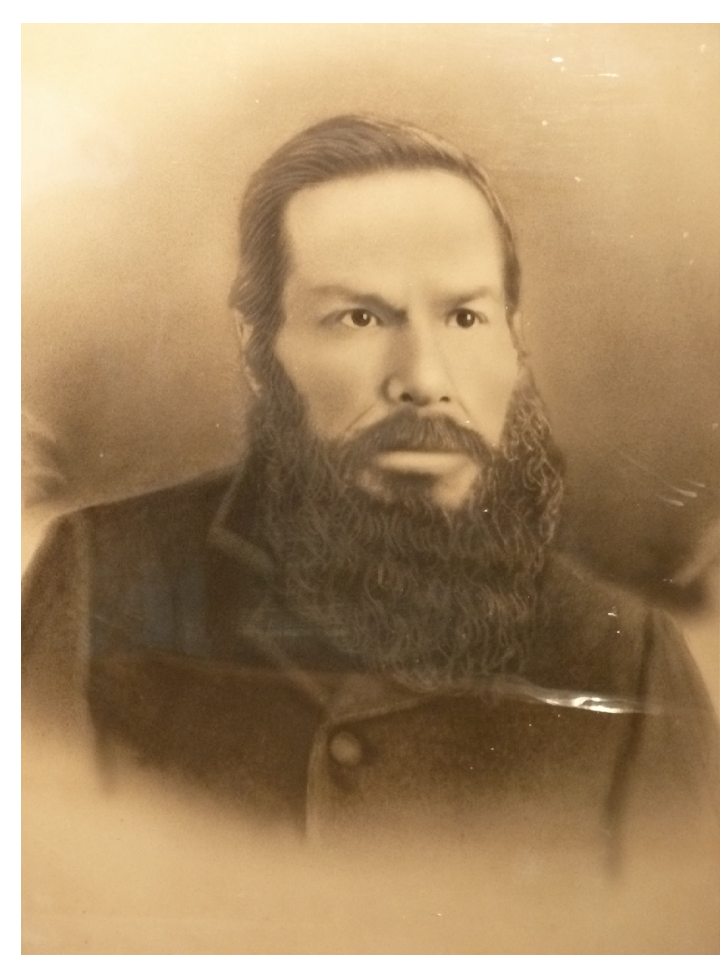

Photo 5 - Éloi Riou, seigneur et maire de Trois-Pistoles (source : famille Rioux)

L'examen du cadastre de 1858 permet cependant de déceler les avantages que possède le seigneur primitif de Trois-Pistoles en comparaison des coseigneurs. En effet, à titre de seigneur principal, Éloi Rioux détient toutes les terres non concédées du fief. Cet élément est non négligeable puisque, selon l'Acte seigneurial de 1854, les terres non concédées au moment de l'abolition deviennent la propriété privée des seigneurs primitifs. Selon Benoît Grenier et Michel Morissette, cet aspect de la loi de 1854 sera lourd de conséquence pour la période suivant l'abolition.

Cette décision de maintenir ces terres non concédées dans le domaine privé a pour effet, à une époque caractérisée par l'urbanisation et l'industrialisation, de confirmer les seigneurs [...] en tant que grands propriétaires fonciers. Dans les seigneuries de peuplement, les terres non concédées sont de plus en plus rares dans la seconde moitié du XIXe siècle et les domaines donneront fréquemment lieu à des formes variées de mise en valeur à des fins immobilières. Souvent bien situés dans les seigneuries, près de l'église et du noyau villageois, les domaines et autres terres réservées ont dès lors pu faire l'objet de lotissements, en particulier en milieu urbain ou périurbain. Les seigneurs sont alors les mieux placés pour jouer un rôle-clé dans le développement urbain ${ }^{87}$.

L'analyse du cadastre abrégé du district de Québec révèle aussi l'importante disparité qui existe entre la valeur des parts de la seigneurie. Alors que les plus grands propriétaires pos-

87. Benoît Grenier et Michel Morissette, «Propriétés et propriétaires seigneuriaux dans l'Est du Québec entre 1854 et le milieu du XXe siècle : le cheminement comparé de l'île d'Anticosti et de Rivière-du-Loup », dans Harold Bérubé et Stéphane Savard, dir., Pouvoir et Territoire au Québec depuis 1850, Québec, Septentrion, 2017, p. 40-41. 
sèdent des parties de la seigneurie valant plusieurs milliers de dollars, Raphaël Plourde n'a qu'une infime part d'une valeur de trente-trois cents. Cela pousse à réfléchir à ce que signifie réellement être coseigneur à Trois-Pistoles. En plus du fait que vingt-neuf coseigneurs ne possèdent que leur propre terre en fief, la majorité des vingt-quatre autres coseigneurs détiennent seulement de petites parts de la seigneurie. Un individu comme Raphaël Plourde peut-il véritablement mettre à profit son statut de coseigneur afin d'élever sa position sociale ou au contraire le nombre considérable de coseigneurs présents dans le fief a-t-il pour effet de «neutraliser » le prestige lié à la possession de ce statut?

Quoi qu'il en soit, le cadastre abrégé rédigé par le commissaire Siméon Lelièvre en 1858 permet de constater à quel point les partages successoraux successifs peuvent avoir un effet d'émiettement au sein des fiefs laïcs de la vallée du Saint-Laurent. Le travail minutieux réalisé par ce notaire permet également de remettre en question l'influence de la lignée seigneuriale principale au sein du fief puisque celle-ci ne possède même plus au moment de l'abolition la partie la plus significative (financièrement du moins) de la seigneurie.

Maintenant que la situation du fief au moment de l'abolition du régime seigneurial a été scrutée, intéressons-nous à l'état des lieux lors du rachat des rentes seigneuriales par le SNRRS. Cet exercice se révèle cependant plus difficile à effectuer que le précédent, car de nombreux documents du SNRRS sont manquants à propos de Trois-Pistoles. L'examen de la seigneurie au moment du rachat des rentes sera donc partiel et devra se baser sur les documents disponibles. Ces derniers permettent de constater que les rapports du SNRRS portant sur Trois-Pistoles datent du tout début des années 1940 (plus précisément entre 1941 et 1943) ce qui est normal puisque comme le souligne Benoît Grenier, «En 1944, l'essentiel de l'opération sera complétée ${ }^{88} »$. Les documents du Syndicat qui sont disponibles nous apprennent que Joseph Ernest Rioux possède « un titre parfait à la seigneurie de Trois-Pistoles » en ce qui concerne la première partie de la seigneurie ${ }^{89}$. La partie trois de la seigneurie revient quant à elle à Anita Rioux, la partie sept à Charles Rioux, la partie huit à Victor et Benoît Morency, la partie treize à Emelia Bérubé et la partie vingt à Thomas D'Amours ${ }^{90}$. En comparant les noms des propriétaires du début des années 1940 avec ceux des propriétaires du milieu du siècle précédent, il est possible de constater une certaine continuité familiale dans la propriété seigneuriale, malgré certains changements. En effet, la première, la troisième et la septième parties de la seigneurie appartiennent au début des années 1940 aux mêmes familles Rioux qui les possédaient en $1858^{91}$. La partie vingt est également restée dans la même famille puisqu'en 1943, comme en 1858, c'est un membre de la famille D'Amour(s) qui possède cette partie ${ }^{92}$. Cependant, les parties huit et treize du fief ont changé de main entre 1858 et les années 1940. Ces parts appartenant à des Rioux au moment de la confection du

88. Grenier, Brève histoire du régime seigneurial, p. 208.

89. BAnQ-Québec, E39, S100, SS1, Fonds Syndicat national du rachat des rentes seigneuriales, Seigneurie de TroisPistoles.

90. Ibid.

91. Ibid.

92. Ibid. 
cadastre abrégé sont en effet plus de quatre-vingts ans plus tard entre les mains de Benoît Morency et Emelia Bérubé, et ce, en raison de ventes survenues entre-temps ${ }^{93}$.

Outre les continuités et les ruptures au sein des familles propriétaires de parties de la seigneurie, les rapports du SNRRS permettent aussi de mieux comprendre la stratégie mise de l'avant par la famille seigneuriale principale afin d'éviter d'accentuer de façon trop importante le morcellement de sa partie du fief. En effet, lorsque le seigneur Éloi Rioux décède en 1865, il n’a pas préparé de testament, ce qui fait en sorte que son fief est divisé en parts égales entre l'ensemble de ses neufs enfants ${ }^{94}$. Son fils aîné, Joseph-Magloire Rioux, se retrouve alors à posséder seulement $1 / 9$ de la partie de la seigneurie appartenant à sa famille. Mais dans les décennies qui suivent la mort d’Éloi Rioux, les descendants de celui-ci déploient une stratégie de remembrement du «fief » en donnant ou en vendant à JosephMagloire Rioux leur part respective dans la seigneurie. C'est ainsi qu'en 1929, après l'achat de la part de son frère Samuel, Joseph-Magloire Rioux réunifie finalement l'ensemble de I’héritage seigneurial légué par Éloi Rioux à ses neufs enfants ${ }^{95}$. Cette stratégie de remembrement de la seigneurie qui est déployée à la fin du XIXe et au début du XXe siècle par la lignée seigneuriale principale de Trois-Pistoles semble être plutôt inusitée pour cette époque où la tendance est plutôt au « morcellement du bien seigneurial » en raison de « l'absence flagrante de stratégie familiale en ce qui concerne la reproduction seigneuriale ${ }^{96} »$. Anita Rioux, qui est à la fois la fille de Joseph-Magloire Rioux et la dernière seigneuresse de TroisPistoles, ne connaît pas les mêmes difficultés que son père à éviter le morcellement de son héritage puisqu'elle est enfant unique en raison du mariage tardif de ses parents ${ }^{97}$.

En conclusion, l'examen du processus d'abolition du régime seigneurial favorise une meilleure compréhension de la place occupée par la lignée des seigneurs principaux à TroisPistoles. En effet, en plus d'exposer l'important état d'émiettement de la seigneurie, le cadastre abrégé de 1858 permet de mieux comprendre pourquoi l'influence de la dynastie seigneuriale principale peut se voir remise en question dans ce fief où plusieurs coseigneurs possèdent assez de terres ou de censitaires pour concurrencer directement l'hégémonie des seigneurs primitifs au sein du monde seigneurial et paysan. Les rapports fragmentaires du SNRRS permettent quant à eux de mieux saisir la perpétuation de la propriété seigneuriale au sein des familles ayant les plus grandes parts de la seigneurie (c'est-à-dire les deux principales lignées de Rioux) ainsi que la stratégie employée par la lignée seigneuriale principale après l'abolition de 1854 afin de ne pas diviser la propriété seigneuriale encore davan-

93. Ibid. ; Michel Morissette, «Les persistances de l'« Ancien Régime» québécois : seigneurs et rentes seigneuriales après l'abolition (1854-1940) », Mémoire de maîtrise (histoire), Université de Sherbrooke, 2014, 161 p.

94. BAnQ-Québec, E39, S100, SS1, Fonds Syndicat national du rachat des rentes seigneuriales, Seigneurie de TroisPistoles.

95. BAnQ-Québec, E39, S100, SS1, Fonds Syndicat national du rachat des rentes seigneuriales, Seigneurie de TroisPistoles.

96. Benoît Grenier (avec la collaboration de Michel Morissette), « Les persistances de la propriété seigneuriale au Québec ou les conséquences d'une abolition partielle et progressive (1854-1940) », Histoire \& Sociétés rurales, vol. 40 (2 ${ }^{\mathrm{e}}$ trimestre 2013), p. 61-96.

97. Entretien 2015-03 avec Anita Rioux, Trois-Pistoles, 15 mai 2015. 
tage. Cette partie a donc permis de relativiser le pouvoir des seigneurs principaux de TroisPistoles en remettant en perspective leur prédominance réelle au sein de la seigneurie. Mais quelle conséquences concrètes cette réalité a-t-elle sur les traces mémorielles seigneuriales présentes à Trois-Pistoles après 1854 ? C'est ce que nous tâcherons d'examiner grâce à la troisième et dernière partie de cette recherche.

\section{Traces et mémoire de la dynastie seigneuriale des Rioux à Trois-Pistoles}

\section{Une mémoire seigneuriale vivante garante d'une notoriété persistante}

L'abolition du régime seigneurial en 1854 ne marque pas pour la majorité des seigneurs " une rupture radicale » avec l'époque antérieure ${ }^{98}$. En effet, les seigneurs demeurent des acteurs influents dans leur milieu et ils continuent d'entretenir des rapports marqués par l'inégalité sociale avec leurs censitaires puisque ceux-ci continuent pour la majorité à venir payer leurs rentes constituées, le 11 novembre de chaque année, comme ils le faisaient auparavant avec les rentes seigneuriales et le cens. La fonction de seigneur perd par contre de son lustre, notamment en raison de l'abolition des privilèges honorifiques seigneuriaux ${ }^{99}$.

Malgré tout, l'ancienneté de la présence seigneuriale laisse inévitablement des traces mémorielles importantes dans les différentes localités de la vallée du Saint-Laurent. Cette mémoire garante de notoriété permet ainsi aux seigneurs qui le désirent de se positionner favorablement dans la sphère politique locale qui se développe au Québec au milieu du XIXe siècle, car comme le souligne Benoît Grenier "La fonction de maire apparaît comme une continuité logique de celle de seigneur et témoigne de l'absence de rupture au sein du monde rural laurentien au moment où le Parlement met fin à plus de deux cents ans de vie sous le régime seigneurial ${ }^{100} »$. Cette continuation entre la fonction de seigneur et celle de maire est parfaitement illustrée à Trois-Pistoles où le « dernier seigneur » Éloi Rioux est élu au poste de maire en $1855^{101}$. L'élection d'Éloi Rioux à la tête de la municipalité apparaît comme étant révélatrice de la position privilégiée qu'occupe la famille seigneuriale principale à Trois-Pistoles en raison de son histoire. En effet, pour être porté au pouvoir, l'aspirant maire doit non seulement obtenir le suffrage de ses concitoyens, mais aussi avoir une notoriété, un réseau de contacts et un bagage personnel conséquent. Or, la première partie de cette étude a permis d'exposer que tout en étant enraciné au plus près de la population, les Rioux font aussi partie à leur manière de la notabilité locale. Cette position faisant d'eux des gens étant proches à la fois des milieux de pouvoir et des milieux populaires est donc optimale afin de briguer avec succès les postes électifs locaux. II semble toutefois que ce

98. Grenier, Brève histoire du régime seigneurial, p. 191.

99. Ibid., p. 204.

100. Grenier, Seigneurs campagnards, p. 229.

101. Rioux, Histoire de Trois-Pistoles, p. 223. 
rang social leur attirant tout autant le respect de la masse que de l'élite soit largement le fruit d'une construction mémorielle bien enracinée dans l'esprit des habitants de la localité. En effet, durant la période suivant l'abolition, le pouvoir réel d'Éloi Rioux (puis de son fils Joseph-Magloire Rioux) est relatif si l'on prend en considération que ces deux seigneurs (et leur famille) n'ont ni la richesse et l'instruction faisant la force des notables, ni les mêmes pouvoirs que détenaient leurs ancêtres sur les habitants pendant que le régime seigneurial était en place ${ }^{102}$. Il semble donc que l'élection d'Éloi Rioux à titre de premier maire de TroisPistoles soit en bonne partie redevable à la notoriété acquise par la famille seigneuriale Rioux au fil des générations. D’ailleurs, cette même notoriété semble se perpétuer dans les mémoires malgré le passage du temps, car Joseph-Magloire Rioux est lui aussi élu maire de la municipalité durant les premières décennies du XX $X^{e}$ siècle ${ }^{103}$.

Ces élections répétées de seigneurs, loin de constituer des cas isolés témoignant de l'influence de la lignée seigneuriale principale à Trois-Pistoles, sont plutôt symptomatiques de la notoriété persistante de la dynastie seigneuriale ${ }^{104}$. En effet, Anita Rioux, qui hérite du «fief » familial dans les années 1930 en raison de son statut de fille unique de JosephMagloire Rioux, a un parcours qui témoigne du rôle social particulier qu'occupent les « seigneurs » et les « seigneuresses » à Trois-Pistoles après l'abolition de 1854 et même après le rachat des rentes dans les années $1940^{105}$.

102. Grenier, Seigneurs campagnards, p. 88-94 et 235-237.

103. Rioux, Histoire de Trois-Pistoles, p. 223.

104. Benoît Grenier, «Élites seigneuriales, élites municipales : Le pouvoir seigneurial à l'heure de l'abolition », dans Thierry Nootens et Jean-René Thuot (dir.), Les figures du pouvoir à travers le temps : Formes, pratiques et intérêts des groupes élitaires au Québec, XVII'-XXe siècles, Québec, Presses de l'Université Laval (collection Cahiers du CIEQ), 2012, p. 57-64.

105. Entretien 2015-03 avec Anita Rioux, Trois-Pistoles, 15 mai 2015. 


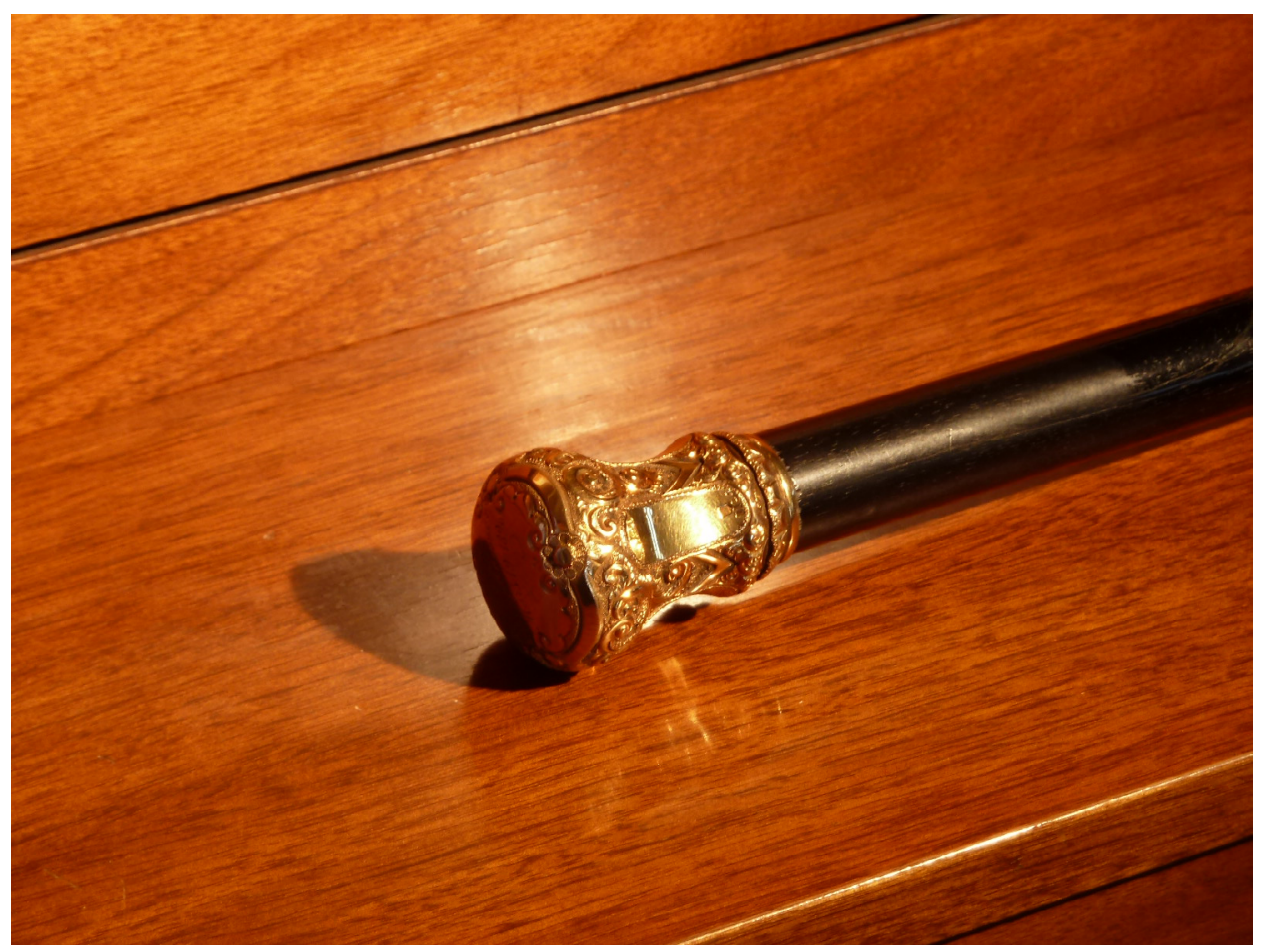

Photo 6 - Canne « seigneuriale » à pommeau d'or de Joseph-Magloire Rioux

Ce rôle singulier s'illustre de multiples façons, mais l'une des plus significatives s'incarne par la fonction de bienfaitrice publique que les habitants de cette municipalité du Bas-SaintLaurent accordent à la « seigneuresse ${ }^{106}$ ». En effet, la communauté pistoloise se tourne régulièrement vers Anita Rioux (à partir du moment où celle-ci prend en main la gestion du patrimoine familial à la suite de la mort de sa mère durant les années 1940) afin de réclamer de celle-ci le don de terres. Comme l'affirme la « seigneuresse », «le maire arrivait puis il nous demandait [un terrain], ça fait qu'on ne refusait pas, on disait toujours oui, [...] ça l'air c'était naturel ${ }^{107}{ }^{\prime}$. Le don de ces terrains qui serviront à accueillir la construction d'une école, d'un cimetière, d'un camping municipal ainsi que d'autres installations publiques expose bien le fait que la population locale considère qu'avec le pouvoir viennent les responsabilités et qu'à ce titre la position privilégiée que les Pistolois consentent à accorder à la famille seigneuriale (notamment en élisant les membres de cette famille au poste de maire) s'accompagne de certains devoirs, dont le soutien au développement local à travers la donation de propriétés foncières ${ }^{108}$. La place singulière occupée par Anita Rioux et sa famille dans la communauté en raison de la continuation d'une mémoire seigneuriale s'exprime aussi par la possession de privilèges symboliques. Parmi ceux-ci se trouve notamment la récitation d'une messe basse annuelle en faveur de la famille seigneuriale et l'obtention de bancs réservés à l'église. Comme le souligne Anita Rioux au sujet de ce dernier point, « Ils

106. Surnom donné à Anita Rioux par les habitants de Trois-Pistoles; Grenier, « Jean Rioux : émigrant breton, seigneur canadien », p. 86.

107. Entretien 2015-03 avec Anita Rioux, Trois-Pistoles, 15 mai 2015.

108. Ibid. 
[les paroissiens] savaient que c'était notre banc et nous autres on payait pas [le banc] $]^{109}{ }$.

Mais le soutien à la communauté et les privilèges honorifiques ne sont pas les seules attributions que les Pistolois décernent à la descendante de la lignée seigneuriale principale puisque la « seigneuresse » est nommée commissaire d'école, puis, présidente de la commission scolaire par sa communauté. En désignant Anita Rioux comme présidente de la commission scolaire, les résidents de Trois-Pistoles et de la région environnante font donc une fois de plus confiance à un descendant de la dynastie seigneuriale afin d'occuper une position de pouvoir. Cette confiance est d'ailleurs séculaire puisque Trois-Pistoles a vu vingt Rioux occuper le poste de président de la commission scolaire depuis la fondation de celleci en $1843^{110}$. Bien qu'ils ne fassent certainement pas tous partie de la lignée seigneuriale principale, ce chiffre prouve indubitablement que les membres de cette famille pionnière possède un crédit important auprès de la population. Cette situation n'est cependant pas unique à Trois-Pistoles, car comme le mentionne Benoît Grenier à propos de la persistance de l'influence seigneuriale dans le Québec des XIX et XX siècles « il est fascinant de constater qu'en l'absence de contraintes la population s'en remet parfois aux anciens maîtres qui s'avèrent des chefs naturels dont on semble accepter naturellement l'ascendant ${ }^{111} »$.

Une autre trace mémorielle du passé seigneurial s'incarne selon les dires de Gaston Rioux, fils d'Anita, à travers la notoriété dont jouissent les Rioux descendants des seigneurs primitifs en raison de leur statut d'héritiers des anciens propriétaires du fief de Trois-Pistoles ${ }^{112}$. Comme le déclare Gaston Rioux:

Le nom Rioux c'est un nom qui est important. Ici, à Trois-Pistoles, on est reconnu comme une famille ancestrale et avec ma mère qui est la seigneuresse, les gens nous regardent d'une autre façon. C'est fascinant de vivre ça. Moi d'ailleurs mon surnom c'est « le seigneur ${ }^{113}$.

Cette position sociale singulière que décrit Gaston Rioux représente un phénomène qui est observable dans plusieurs autres localités de la vallée laurentienne où la perpétuation d'une mémoire seigneuriale se caractérise par «la survivance d'un statut distinctif informel pour les anciens seigneurs » ayant des conséquences «sur les plans social, économique et symbolique » de la vie locale $e^{114}$. À Trois-Pistoles, ce «statut distinctif informel » semble constituer une réalité qui est ressentie tant par les anciens censitaires que par les anciens seigneurs, car les résidents de la municipalité portent aux postes de responsabilité de façon répétée les descendants de la lignée seigneuriale principale tandis que lorsque l'on demande à Gaston Rioux ce que cela représente pour lui d'être le descendant d'une famille seigneuriale, il ré-

109. Ibid.

110. Rioux, Histoire de Trois-Pistoles, p. 603.

111. Grenier et Morissette, «Propriétés et propriétaires seigneuriaux dans l'Est du Québec », p. 52.

112. Comme le mentionne Mme. Anita Rioux durant son entretien, elle a épousé un Rioux, ce qui explique que son fils Gaston porte lui aussi le nom « Rioux ».

113. Entretien 2015-04 avec Gaston Rioux, Trois-Pistoles, 15 mai 2015.

114. Grenier, Brève histoire du régime seigneurial, p. 213. 
pond sans hésiter « C'est de la notoriété pour moi!115 ».

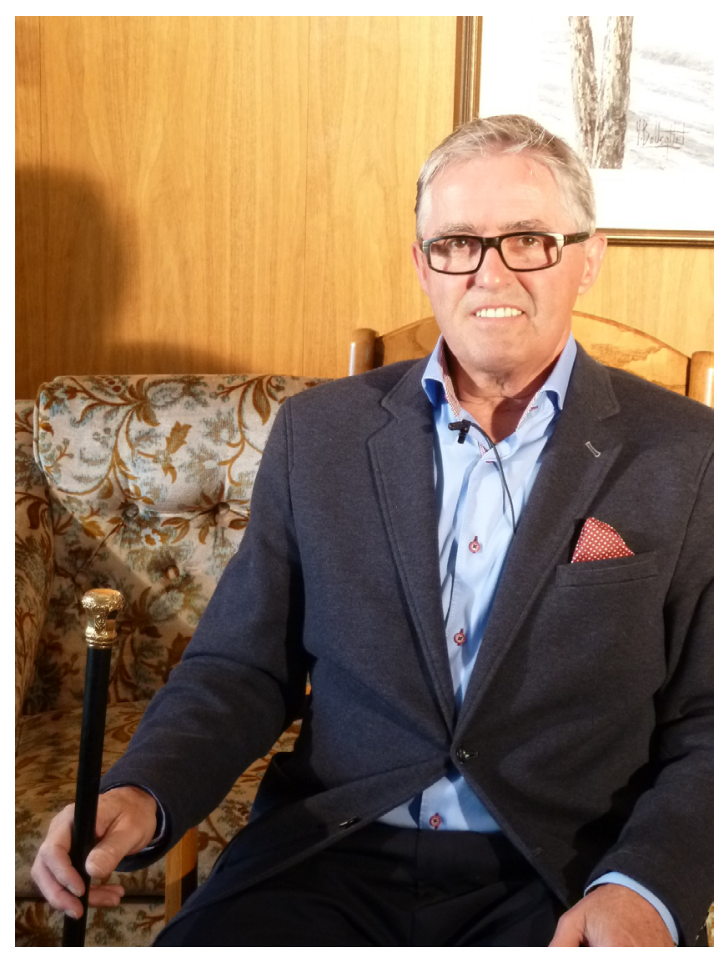

Photo 7 - Gaston Rioux, surnommé le « seigneur »

\section{Un patrimoine seigneurial qui ne s'oublie pas}

À Trois-Pistoles, la persistance d'un patrimoine seigneurial malgré l'abolition de 1854 est un fait indéniable. Les traces du régime seigneurial sont observables dans de nombreux domaines dont la propriété foncière, le patrimoine bâti et la toponymie. Tout d'abord, l'analyse croisée du témoignage de Gaston Rioux et du texte rédigé par Benoît Grenier et Michel Morissette permet de constater à quel point la conservation du domaine et des terres non concédées par la famille seigneuriale après l'abolition est un élément primordial dans la persistance de la mémoire seigneuriale dans les localités québécoises. Lorsque Gaston Rioux décrit la localisation des terres héritées de la seigneurie qui sont toujours en possession de sa mère dans la seconde partie du XXe siècle, il évoque un ensemble foncier situé « ici, autour de Trois-Pistoles » en spécifiant qu' «à l'ouest ça se limitait au ruisseau Renouf, au sud vers le deuxième rang ou troisième rang et à l'est on se rendait à l'église ${ }^{116}$ ». L'analyse géographique du territoire ainsi délimité par Gaston Rioux permet de constater facilement que les terres possédées par Anita Rioux et ses ancêtres entre l'abolition du régime seigneurial et la fin du XXe siècle sont situées exactement où se trouve le noyau urbain de Trois-Pistoles. Cette mainmise sur les terres qui reçoivent au fil des ans l'étalement urbain de la municipalité n'est pas sans rappeler le cas des Fraser à Rivière-du-Loup. En effet, cette famille seigneuriale qui « possède un immense domaine au centre de la seigneurie avant l'abolition »,

115. Entretien 2015-04 avec Gaston Rioux, Trois-Pistoles, 15 mai 2015.

116. Ibid. 
voit sa propriété foncière devenir une source de richesse au cours de la fin du XIXe siècle et du début du siècle suivant au fur et à mesure que les propriétés terriennes héritées de la seigneurie sont sollicitées afin d'accueillir le développement urbain de la municipalité de Rivière-du-Loup ${ }^{117}$. Cette possession des terres les mieux situées donne aux Fraser le « statut de grands propriétaires fonciers » et leur assure une position d' " acteurs incontournables du développement de la ville de Fraserville [...] au moins jusqu'en 1950118 ». Cette situation est certainement analogue avec celle vécue par les Rioux, quoique les anciens seigneurs de Trois-Pistoles ont sans doute profité d'une situation un peu moins favorable que les Fraser en raison de l'émiettement considérable de leurs propriétés et de la croissance moins importante de leur ville. Il n'empêche que ces deux familles seigneuriales s'illustrent par des stratégies fort semblables en ce qui concerne la gestion de leur patrimoine foncier subsistant après l'abolition de 1854. En effet, les Fraser et les Rioux ont tous deux recours à des baux emphytéotiques afin de distribuer leurs terres selon des modalités perpétuant la relation de propriétaire-locataire caractérisant le régime seigneurial ${ }^{119}$. D'ailleurs, la fin des locations par baux emphytéotiques n'a lieu à Trois-Pistoles qu'au tournant du XXIe siècle.

La place centrale qu'occupe selon toute vraisemblance les descendants des seigneurs Rioux dans « la gestion et [...] l'aménagement du territoire urbain » donne donc au patrimoine seigneurial de cette famille une existence tangible puisqu'il continue d'influencer la vie quotidienne des Pistolois jusqu'à la seconde moitié du XX $X^{e}$ siècle ${ }^{120}$. Cet état des choses favorise donc la perpétuation d'une mémoire seigneuriale locale, l'influence des « seigneurs » Rioux n'étant pas seulement un souvenir historique, mais également un élément observable pour tous les résidents de Trois-Pistoles pendant plus d'un siècle après l'abolition du régime seigneurial en 1854. Cette réalité est bien illustrée par Gaston Rioux lorsqu'il affirme « on était reconnu pour être une famille riche, on était riche en terres, pas riche en argent [...] les gens s'adressaient toujours au seigneur pour avoir un terrain ou un lopin de terre, c'était le seigneur qui était comme le dirigeant de la ville ${ }^{121} »$.

Le patrimoine bâti constitue une autre trace mémorielle du régime seigneurial qui persiste à Trois-Pistoles. En effet, plusieurs bâtiments de la localité rappellent le souvenir de l'époque seigneuriale et des seigneurs Rioux. D’abord, le Manoir Rioux-Belzile, construit au XVIII siècle pour accueillir la famille du seigneur primitif, se dresse toujours à Trois-Pistoles. Cet immeuble constituant un constant rappel du passé seigneurial de la famille Rioux est d'autant plus significatif qu'il est l'un des plus vieux bâtiments de la municipalité ${ }^{122}$.

La maison seigneuriale représente un autre bâtiment perpétuant la mémoire des seigneurs

117. Grenier et Morissette, «Propriétés et propriétaires seigneuriaux dans l'est du Québec », p. 53.

118. Ibid., p. 60.

119. Ibid., p. 55.

120. Ibid., p. 58.

121. Entretien 2015-04 avec Gaston Rioux, Trois-Pistoles, 15 mai 2015.

122. Rioux, Histoire de Trois-Pistoles, p. 578. ; Robert Larin et Marie-Joëlle Larin-Lampron, Le manoir Rioux-Belzile à Trois-Pistoles, Québec, Septentrion, 2013, 256 p. 
Rioux dans cette localité du Bas-Saint-Laurent. L'immeuble ancestral a été déménagé à deux reprises, mais se trouvait initialement en bordure du chemin du Roi sur les rives du fleuve Saint-Laurent. Toujours habité par des descendants des seigneurs Rioux, elle incarne la continuité et l'enracinement de cette famille à Trois-Pistoles ${ }^{123}$. Gaston Rioux considère d'ailleurs que ce bâtiment constitue l'une des traces les plus concrètes du passé seigneurial de Trois-Pistoles. Il souligne en ce qui concerne cette maison « elle appartient à ma sœur et c'est un bien que je ne laisserais pas partir en dehors de la famille ${ }^{124}$ ». Mais son attachement envers ce bien patrimonial et familial n'empêche pas le fils d'Anita Rioux d'être lucide quant à l'espace mémoriel réel qu'occupe cette maison dans l'esprit des Pistolois. En effet, selon lui, «seulement les personnes d'un certain âge » connaissent le passé seigneurial de la demeure ${ }^{125}$.

La toponymie constitue une autre forme sous laquelle se véhicule la mémoire seigneuriale à Trois-Pistoles. L'une des deux rues principales de la ville se nomme en effet Jean-Rioux, et ce en hommage à ce seigneur pionnier de Trois-Pistoles ${ }^{126}$. De plus, les belvédères situés sur l'île aux Basques portent également le nom de ce seigneur-défricheur et de son épouse Catherine Leblond ${ }^{127}$. Le fait que le seul seigneur ayant droit à un hommage dans la toponymie locale soit Jean Rioux porte à croire que c'est surtout en raison de son rôle de pionnier des lieux qu'il est honoré, bien qu'il ne faille pas non plus sous-estimer sa fonction seigneuriale. L'absence des autres seigneurs de Trois-Pistoles dans la toponymie est cependant un élément qu'il faut souligner puisque cela témoigne d'un focus mémoriel à prime abord difficilement explicable en raison de l'influence importante occupée par d'autres seigneurs dans la vie locale (pensons notamment à Éloi Rioux qui fut le premier maire élu de la localité). La rue de la Seigneurie est un autre rappel du passé seigneurial au sein de la toponymie locale. L'emplacement de cette rue n'est pas anodin puisqu'elle débouche sur « ce qui reste de la seigneurie Rioux de Trois-Pistoles », c'est-à-dire sur la dernière propriété émanant du domaine ou des terres non concédées des Rioux qui soit toujours en possession de la famille seigneuriale ${ }^{128}$. Le fait que la rue ait été nommée ainsi expose bien la sensibilité de la municipalité vis-à-vis du patrimoine seigneurial et de la place des Rioux dans l'histoire de Trois-Pistoles. En effet, puisque la rue donne accès à des terres appartenant aux descendants des seigneurs Rioux, cette voie d'accès aurait pu être nommée selon le nom de l'un des seigneurs de la famille ou tout simplement «rue Rioux». En nommant ainsi cette rue, la Ville expose le lien intrinsèque établi dans l'esprit des Pistolois entre le régime seigneurial et la famille Rioux, un lien tout à fait compréhensible dans l'optique où cette famille a régné sur ce fief durant presque la totalité de l'existence de celui-ci.

123. Ibid.

124. Entretien 2015-04 avec Gaston Rioux, Trois-Pistoles, 15 mai 2015.

125. Ibid.

126. Ibid.

127. Ibid

128. Ibid. 


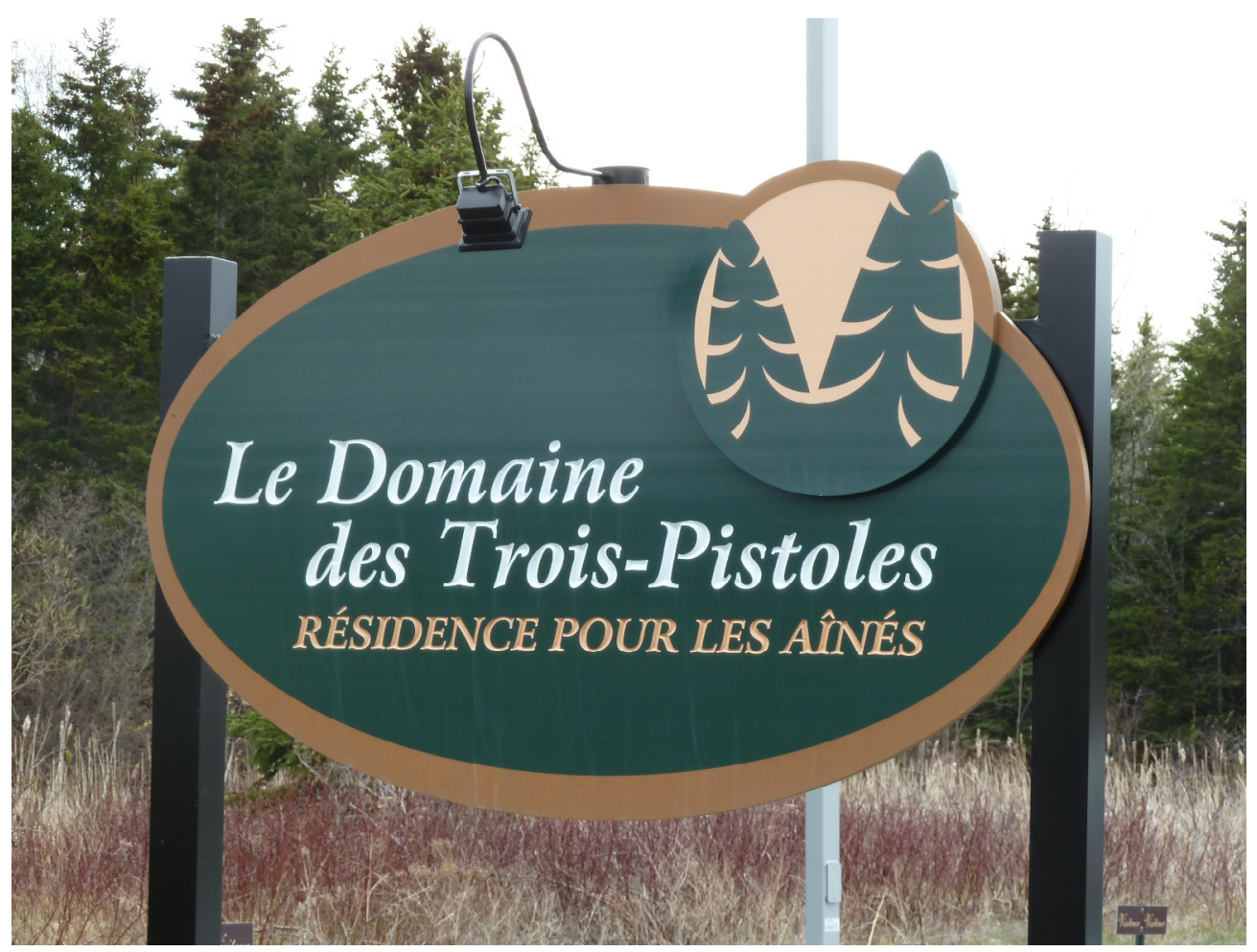

Photo 8 - Au cœur de l'ancien « domaine » seigneurial.

Cette troisième et dernière partie a donc été l'occasion d'examiner la mémoire seigneuriale présente à Trois-Pistoles depuis l'abolition du régime seigneurial en 1854. Dans un premier temps, l'examen du rôle occupé par la famille seigneuriale dans la communauté a permis de constater que l'abolition ne constitue pas nécessairement un moment de rupture complète pour les descendants des seigneurs, mais davantage le début d'une période de transformation où leur rôle évolue pour devenir de plus en plus politique. L'occupation répétitive de postes électifs par les membres de la lignée seigneuriale principale est ainsi un témoignage de la perpétuation d'une mémoire seigneuriale au sein de laquelle les Rioux sont considérés comme des leaders traditionnels dont la place naturelle est d'être aux postes d'influence de la localité. En second lieu, l'étude du patrimoine seigneurial a permis de mieux comprendre comment la mémoire seigneuriale a survécu à Trois-Pistoles. L'analyse du domaine foncier de la famille seigneuriale ainsi que des bâtiments et de la toponymie reliée à celle-ci a mis en lumière la présence de plusieurs éléments concrets de persistance seigneuriale dans cette municipalité. Les réminiscences du régime seigneurial continuant à avoir un impact sur leur vie tout au long des XIXe et XXe siècles, les citoyens de la municipalité semblent avoir préservé un souvenir du passé seigneurial relativement vif, et ce au moins jusqu'à la seconde moitié du XX siècle. Aujourd'hui, la mémoire seigneuriale s'efface progressivement au fur et à mesure où les personnes ayant déjà payé des rentes constituées ou acheté des terres à la « seigneuresse » quittent ce monde. Cependant, le souvenir des seigneurs Rioux semble refuser de mourir complètement comme nous le verrons en conclusion. 


\section{Conclusion}

Cette étude visait à approfondir nos connaissances sur l'histoire et la mémoire du fief de Trois-Pistoles et de la famille seigneuriale Rioux. Pour commencer, nous avons cherché à comprendre comment cette famille seigneuriale d'humble extraction a pu conserver son fief jusqu'à l'abolition du régime seigneurial alors que la vaste majorité des autres familles de même condition n'ont pas réussi. Leur enracinement dans leur milieu et leur communauté, la densité de leur réseau familial ainsi que leur insertion dans la notabilité qui s'accompagne de la préservation d'une position privilégiée auprès de la paysannerie sont les principaux facteurs ayant été relevés afin d'expliquer la durabilité hors norme de cette modeste famille à la tête de son fief.

Dans la deuxième section de cette recherche, nous avons tenté d'éclaircir le déroulement de l'abolition du régime seigneurial à Trois-Pistoles. Après avoir brièvement exposé les raisons poussant le pouvoir politique à abolir ce régime datant de l'époque féodale, nous avons étudié le cadastre abrégé portant sur Trois-Pistoles qui fut réalisé au moment de l'abolition. Cette analyse a révélé plusieurs faits pertinents, dont le considérable état de morcellement dans lequel se trouve le fief au milieu du XIXe siècle et la concurrence importante que subit le seigneur « primitif» de la part de certains autres coseigneurs. Par la suite, l'examen des rapports du SNRRS a permis de constater que la possession des rentes constituées semble avoir connu une continuité en demeurant entre les mains des mêmes familles dans la majorité des cas observables.

Finalement, la dernière partie de notre recherche a été l'occasion de nous pencher sur la mémoire seigneuriale présente à Trois-Pistoles après 1854. Nous avons d'abord observé que la notoriété importante que détiennent les descendants des seigneurs est étroitement liée à l'empreinte mémorielle qui a été laissée par les Rioux au fil des années et que cette notoriété a permis aux membres de la famille de briguer avec succès de nombreux postes électifs locaux. Cette présence non négligeable des Rioux dans les positions d'influence est ainsi devenue un élément favorisant la perpétuation d'une mémoire seigneuriale dans la localité bien après l'abolition de 1854. Par la suite, l'analyse du patrimoine seigneurial ayant résisté à l'abolition a démontré l'importance joué par le patrimoine foncier des Rioux dans la conservation d'une mémoire seigneuriale à Trois-Pistoles. Le patrimoine bâti et la toponymie se sont quant à eux révélés être des éléments d'une importance relative dans la persistance seigneuriale, malgré l'importance de ces éléments pour la famille seigneuriale ellemême. Bref, les hypothèses formulées au commencement de cette réflexion ont toutes été confirmées, mais la réalisation de la recherche a eu pour conséquence d'en faire émerger de nouvelles qui gagneraient à être étudiées plus en profondeur. 
Au terme de cette étude portant sur les persistances seigneuriales présentes à Trois-Pistoles, il nous semble légitime de livrer notre interprétation sur la spécificité de la mémoire seigneuriale locale. Tout d'abord, la mémoire seigneuriale qui s'est transmise dans cette municipalité semble très sélective puisqu'elle est presque complètement axée autour de la famille seigneuriale, et ce au détriment du souvenir du régime seigneurial lui-même. Cette situation n'est cependant pas étonnante, car comme le rappel Tzvetan Todorov, «la mémoire [...] est forcément une sélection : certains traits de l'événement seront conservés, d'autres sont immédiatement ou progressivement écartés, et donc oubliés ${ }^{129}$ ». De l'époque seigneuriale, les Pistolois ont donc gardé en mémoire les rôles de leaders locaux occupés de façon séculaire par les seigneurs Rioux. Cette persistance mémorielle spécifique n'est certainement pas étrangère à la position de prépondérance qu'ont occupé subséquemment à l'abolition de 1854 les seigneurs et leurs descendants. Mais la mémoire seigneuriale présente aujourd'hui à Trois-Pistoles en est-elle pour autant plus importante qu'ailleurs dans la vallée du SaintLaurent? Cette question est délicate, car il faudrait minimalement analyser un échantillon conséquent de seigneuries québécoises afin de pouvoir dresser avec certitude une comparaison de ce type. Cependant, le fait que les descendants directs des seigneurs vivent toujours en grand nombre à Trois-Pistoles, que certains d'entre-eux se font encore surnommer « le seigneur » et que la dernière seigneuresse des lieux pouvait encore y être vue il y a quelques mois à peine constituent autant d'éléments qui nous poussent à affirmer que la persistance seigneuriale présente à Trois-Pistoles atteint un niveau significativement plus élevé que dans la majorité des autres localités ayant un passé seigneurial ${ }^{130}$.

129. Tzvetan Todorov, Les abus de la mémoire, Paris, Les Éditions Arléa, 1995, p. 14.

130. Entretien 2015-04 avec Gaston Rioux, Trois-Pistoles, 15 mai 2015. 